\title{
Rise of the digital machine
}

\section{Genomes and language suggest that biological and social complexity emerge from how information is used, argues Mark Pagel, not from how much of it there is.}

\begin{abstract}
Mark Pagel
Human societies and multicellular organisms share a puzzling feature. They seem to be under-specified. Our societies depend on many more interactions among group members than there are members. Multicellular organisms have many more parts, and connections among those parts, than they have genes. This points to a principle of regulation in the evolution of such complex adaptive systems: complexity arises not from the number of genes or actors but from how those elements are expressed or deployed.
\end{abstract}

This simple conjecture can explain some remarkable anomalies. Humans are almost unimaginably complex, with trillions of cells organized into hundreds of different tissues. But we have scarcely more genes than a fruitfly or a worm, and only about four or five times as many as brewers' yeast or some bacteria. Surprising then that the human genome is 250 times larger than the yeast's. It comprises about 99\% 'junk DNA' genetic code that is not used to make the protein building blocks of life.

Junk DNA gives every appearance of fulfilling the metaphor of the selfish gene. It accumulates in organisms' genomes simply because it is good at accumulating; it can even be harmful. Why we put up with it has long been a mystery.

Increasingly, it seems that the genes that do code for proteins may recruit some or all of this junk DNA to regulate when, where and how much they are expressed. Because nearly every cell in the body carries a complete copy of the genome, something has to tell the genes that make eyes not to switch on in the back of the head, or genes for teeth to stay silent in our toes. Something has to instruct genes to team up to produce complex structures such as hearts and kidneys, or the chemical networks that create our metabolism and physiology.

Genes, in effect, use regulation to promote their interests within the bodily phenotype: it is how they vary their exposure to the outside world. Regulation is how we can have over $98.5 \%$ similarity to chimpanzees in the sequences of our coding genes, yet differ so utterly from them.

Indeed, the huge quantity of junk DNA in the genomes of most complex organisms may act as a vast digital regulatory mechanism. Until recently many common machines, such as aeroplanes, clocks, and

even computers were analogue devices, regulated by levers, springs, heat or pressure. Aeroplanes were flown with a stick, springs drove clocks. Digital regulation - instructions encoded in strings of binary numbers arbitrarily long, and hence precise - enabled complexity to increase. Stealth fighters and space shuttles are so complex that they can be flown only by digital

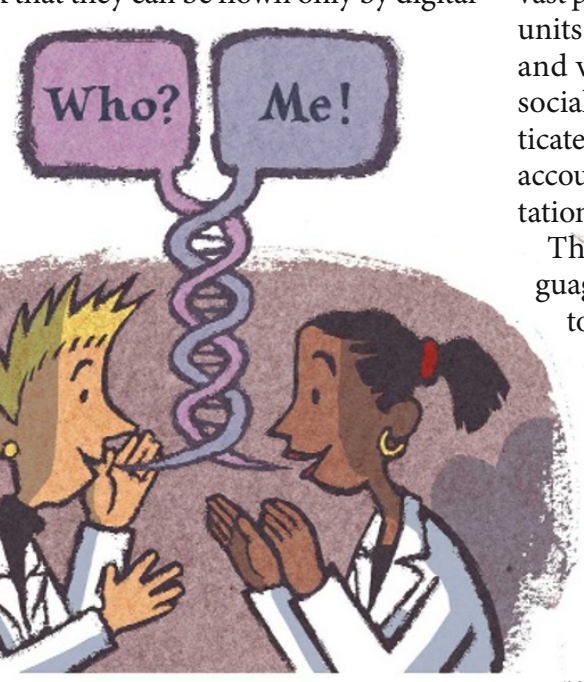

more than the analogue communication systems available to other animals, such as grunts, chest thumping, head butting, odours and bright colours. We need to account for who has done what to whom, when, where and how much they did it, and for what purpose or gain. Human language is a digital communication system of vast possibilities, with words as the discrete units. I suggest it evolved to allow precise and varied regulation of self-interested social behaviour. The exchange of sophisticated verbal information arose to convey accounts of our own and others' acts, reputations, alliances and dues.

The common-sense view is that language arose to promote communication, to coordinate group effort, and to share

knowledge. But if the view put forward here is right, language should deliver more of its value from aiding us to manipulate, distort, bewitch and collude than from the cooperative exchange of information. Ants can manage sophisticated societies and deploy large armies, but only we derive substantial benefits from relying on the goodwill of strangers.

computers, not (analogue) human pilots.

Similarly, the emergence of digital regulation derived from unused stretches of junk DNA may have precipitated the transition from single cells to complex multicellular organisms. Long runs of the four chemical bases that make up DNA can easily act like binary strings. How these stretches bind to a gene can regulate exquisitely the degree and timing of that gene's expression. Tellingly, bacteria and some other single-celled organisms have negligible amounts of junk DNA. They rely far more on analogue systems of gene regulation that are proteinbased and less precise.

A different kind of digital regulation may have made possible the transition to another complex phenotype. Human societies are unique in supporting elaborate systems of cooperation and exchange among unrelated individuals. We help others we may never see again, and we can operate a division of labour - while some hoe the earth, others fetch water. The specialization that cooperation allows is almost certainly responsible for humans' rapid spread out of Africa and around the world. But this kind of social system abounds with ways for cheats to exploit cooperators.

Regulating the social complexity of human societies, then, required something
This depends on how our community judges us and others - verbally.

We use language to promote our interests in society: it is how we vary our expression in the complex social phenotype. As language became more sophisticated in our ancestors it presented more opportunities for selfish gain. We embroider and exaggerate our own dossiers and gently diminish or disparage those of others. We alter our speech or dialect strategically — linguists call it 'code-switching' - to advertise our conformity to particular groups. We can even employ language to enforce moral norms that proscribe or regulate others' social behaviour. Deep down, language may be just the latest form of gene regulation - the voice of our genes. Information management, not lots of parts, is the key to complexity.

Mark Pagel is in the School of Biological

Sciences, University of Reading, Reading RG6 6AJ, UK, and the Santa Fe Institute, 1399 Hyde Park Road, Santa Fe, New Mexico, USA 87501.

\section{FURTHER READING}

Encode Project Consortium Nature 447, 799-815 (2007)

Gell-Mann, M. The Quark and the Jaguar: Adventures in the Simple and Complex (W. H. Freeman, San Francisco, 1994).

Mattick, J. S. BioEssays 25, 930-939 (2003).

Nowak, M. A. \& Sigmund, K. Nature 437, 1291-1298 (2005)

\section{$\frac{n}{\frac{n}{v}}$}

